\title{
Microsymposia
}

MS.77.1

Acta Cryst. (2008). A64, C131

\section{Diffuse scattering as a probe of local structure}

Richard Welberry, Darren J Goossens, Andrew G Beasley, Eric J Chan, Aidan P Heerdegen

Australian National University, Research School of Chemistry, Research School of Chemistry, Australian National University, Acton, Canberra, ACT, 0200, Australia, E-mail:welberry@rsc.anu.edu.au

The strong sharp Bragg reflections that occur in diffraction patterns of all real crystals are used by conventional X-ray crystallography to deduce the average repetitive arrangements of atoms or molecules. Diffuse scattering, on the other hand, contains information about the deviations from the average (i.e., different types of disorder) and gives structural information on a scale that goes beyond that of the average unit cell and extends over a range of $0.1 \mathrm{~nm}-100 \mathrm{~nm}$. In many important materials, it is this extended range of structural information that is crucial in determining the unique or novel properties of the material, rather than the average unit cell structure. We review the development of our diffuse scattering methodology using a variety of examples taken from different fields to illustrate the kind of information that can be obtained by current day methods information that is simply not available from the Bragg experiment. Amongst these will be the zeolite mordenite, the organic pentachloronitrobenzene, which is one of a series of chloronitrobenzene compounds whose anomalous dielectric properties are thought to be related to their disorder, the relaxor ferroelectric PZN and finally examples of organic molecular crystals that exhibit polymorphism - a topic of crucial interest to the pharmaceutical industry. We gratefully acknowledge the support of the Australian Research Council, the Australian Synchrotron Research Program, the Access to Major Research Facilities Program and the Australian Partnership for Advanced Computing.

Keywords: diffuse scattering, Monte Carlo simulation, local structure

\section{MS.77.2}

Acta Cryst. (2008). A64, C131

\section{Magnetic short-range order in Pt-rich Pt-Mn alloys}

Miwako Takahashi ${ }^{1}$, Timbangen Sembiring ${ }^{2}$, Toetsu Shishido ${ }^{3}$, Yukio Noda ${ }^{4}$, Ken-ichi Ohshima ${ }^{1}$

${ }^{1}$ University of Tsukuba/Japan, Institute of Materials Science, 1-1-1 Tennoudai, Tsukuba, Ibaraki, 305-8573, Japan, ${ }^{2}$ Department of Physics, Faculty of Mathematics and Natural Science, University of Sumatera Utara, Medan, Indonesia., ${ }^{3}$ Institute for Materials Research, Tohoku University, Sendai 980--8577, Japan, ${ }^{4}$ Institute of Multidisciplinary Research for Advanced Materials, Tohoku University,Sendai 980--8577, Japan, E-mail:takahasi@bukko.bk.tsukuba.ac.jp

The magnetism of $\mathrm{Pt}_{(1-\mathrm{c})} \mathrm{Mn}_{\mathrm{c}}$ between $\mathrm{c}=0.12$ to 0.16 is very unique. The magnetic diffraction pattern shows drastic change with a little difference of atomic arrangement and Mn concentration, while magnetic susceptibility shows a spin-glass like behavior throughout the concentration region. The atomic ordered phase in this $\mathrm{Mn}$ concentration range is also unique: it possesses two kinds of atomic ordered structures, $\mathrm{Cu}_{3} \mathrm{Au}$-type as a high temperature phase and $\mathrm{ABC}_{6}$-type as a low temperature phase. The latter structure has the largest unit cell in the fcc based ordered structures and is only found in Pt-rich Pt-Mn, Pt-rich Pt-Cu and ternary $\mathrm{CuMnPt}_{6}$ alloys. In the neutron diffraction pattern of the alloy with $\mathrm{c}=0.125$, diffuse scattering appears at 1/2,0,0 with fourfold incommensurate peaks. The fourfold peaks also appear at equivalent points in the $\mathrm{ABC}_{6}$-type structure. The origin of the incommensurate peaks is considered to be a distant interaction through a spin density wave in the conductionelectron gas caused by a Fermi-nesting effect. In the alloy with $\mathrm{c}=0.144$, the incommensurate peaks disappear completely and a new set of magnetic superlattice reflections appears at 1/2 1/4,0,1/2,0,1/4 and their equivalent points in the $\mathrm{ABC}_{6}$-type structure. These magnetic peaks indicate that the alloy forms long-rang order of typeII anti-ferromagnetic structure. The origin of the magnetic long-range order is considered to be an antiferromagnetic interaction between the near-neighbor localized spins. These characteristic magnetic scattering disappear completely when the alloys become disordered state. The results indicate a strong coupling between the magnetic and atomic structures and a crossover of dominant magnetic interaction in this Mn concentration region.

\section{Keywords: magnetic ordering, neutron diffuse scattering,} spin glasses

\section{MS.77.3}

Acta Cryst. (2008). A64, C131

\section{Phason diffuse scattering in the icosahedral quasicrystalline phases $\mathrm{Zn}-\mathrm{X}-\mathrm{Sc}, \mathrm{X}=\mathrm{Co}, \mathrm{Ag}, \mathrm{Mg}$}

Sonia MP Francoual ${ }^{1}$, Marc De Boissieu ${ }^{2}$, Roland Currat ${ }^{3}$, Shiro Kashimoto ${ }^{4}$, Tsutomu Ishimasa ${ }^{4}$

${ }^{1}$ National High Magnetic Field Laboratory, Los Alamos National Laboratory, sfranco@lanl.gov, Los Alamos, New Mexico, 87545, USA, ${ }^{2}$ Laboratoire de Thermodynamique et Physico-Chimie Metallurgiques, St Martin d'Heres, France, ${ }^{3}$ Institut Laue Langevin, Grenoble, France, ${ }^{4}$ Graduate School of Engineering, Hokkaido University, Sapporo, Japon, E-mail:sfranco@lanl.gov

According to the hydrodynamic theory of quasicrystalline materials, the long range quasiperiodic order yields in quasicrystals new longwavelength modes, the phason modes, in addition to the acoustic phonons. Phason excitations are diffusive and associated with internal atomic rearrangements the dynamics of which is expected to lead to a distinctive diffuse scattering signal around Bragg peaks in reciprocal space. Up to now, experimental demonstration for the existence of phason diffuse scattering was given in the enlightening single case of the Al-Pd-Mn Mackay-type icosahedral (i-) phase from which the phason elastic constants were extracted and the thermal activation unvealed. In the present communication, we report on $\mathrm{X}$-ray synchrotron investigations of the diffuse scattering in the $\mathrm{i}-\mathrm{Zn}-\mathrm{X}-\mathrm{Sc}(\mathrm{X}=\mathrm{Mg}, \mathrm{Ag}, \mathrm{Co})$ phases and the $\mathrm{Zn}-\mathrm{Sc} 1 / 1$ periodic approximant bringing evidence for the presence of long-wavelength phasons in this new class of icosahedral quasicrystalline phases and ruling it out in the

1/1 approximant structure.

Keywords: diffuse X-ray scattering, quasicrystals, phase transitions and structure

\section{MS.77.4}

Acta Cryst. (2008). A64, C131-132

\section{D-PDF analysis of single crystal diffuse scattering on the example of disordered quasicrystals}

Thomas Weber, Philippe Schaub, Walter Steurer

ETH Zurich, Department of Materials, Wolfgang-Pauli-Str. 10, Zurich, Zurich, 8093, Switzerland, E-mail :thomas.weber@mat.ethz.ch

The three-dimensional pair distribution function (3D-PDF) method 


\section{Microsymposia}

is an extension of the widely used powder diffraction based 1D-PDF technique. It is obtained by Fourier transformation of single crystal (diffuse) scattering. Contrary to $1 \mathrm{D}-\mathrm{PDF}$ it provides not only information about the length, but also about the spatial orientation of real inter-atomic vectors. The 3D-PDF method allows employing techniques that are difficult to apply in the case of powder diffraction. Complexity of 3D-PDF maps may be strongly reduced by filtering out information that is either already known or not of interest. As an example Bragg scattering from the usually well-known average structure may be eliminated before calculating the 3D-PDF what strongly enhances visibility of disorder information [1]. Further, independent disorder phenomena may be separated in reciprocal space, if they show distinct sets of diffuse scattering, and/or in PDF space, if the orientation of inter-atomic vectors allows distinguishing different kinds of disorder e.g. intra- and interlayer disorder. An instructive example for demonstrating the power of 3D-PDF analysis is diffuse scattering from quasicrystals, which certainly belongs to the most complex disorder problems. It will be shown that the 3D-PDF method allows understanding of extremely complicated diffuse scattering from decagonal $\mathrm{Al}-\mathrm{Cu}-\mathrm{Co}$ and $\mathrm{Al}-\mathrm{Co}-\mathrm{Ni}$ quasicrystals by multiple reduction of complexity in reciprocal and PDF space $[2,3]$. References:

[1] Ph. Schaub, Th. Weber and W. Steurer, Phil. Mag. 87, 2781, (2007).

[2] M. Kobas, Th. Weber and W. Steurer, Phys. Rev. B 71, paper No. 224205, (2005).

[3] $\mathrm{Ph}$. Schaub, Th. Weber and W. Steurer, in preparation.

Keywords: pair distribution function, diffuse scattering, quasicrystals

\section{MS.77.5}

Acta Cryst. (2008). A64, C132

\section{Nanostructure of silver-free photochromic glasses studied by anomalous small angle $X$-ray scattering}

Sylvio Haas $^{1}$, Andre Heinemann ${ }^{2}$, Rainer Kranold ${ }^{3}$,

Dragomir Tatchev ${ }^{1}$, Guenther Goerigk ${ }^{4}$, Matthias Mueller ${ }^{5}$, Armin Hoell

${ }^{1}$ Hahn-Meitner-Institute Berlin, Glienicker Str. 100, Berlin, Berlin, 14109, Germany, ${ }^{2}$ Institute of Materials and Engineering Science, PMB 1, Menai NSW, Australia, ${ }^{3}$ University of Rostock, Institute of Physics, D-18051 Rostock, Germany, ${ }^{4} \mathrm{FZ}$ Juelich, IFF, JCNS-FRMII, c/o TU-Munich, Lichtenbergstr. 1, D-85747 Garching, Germany, ${ }^{5}$ Otto-Schott-Institute, Friedrich-Schiller-University Jena, Frauenhoferstr. 6, D-07743 Jena, Germany, E-mail:sylvio.haas@hmi.de

Cuprous halide nanocrystals, embedded in a glassy matrix are of interest with respect to their photochromic behaviour. A silverfree photochromic glass was prepared using a $14.2 \mathrm{Na}_{2} \mathrm{O}-6.0 \mathrm{Al}_{2} \mathrm{O}_{3}-$ $26.6 \mathrm{~B}_{2} \mathrm{O}_{3}-53.2 \mathrm{SiO}_{2}$ base glass doped with small amounts of $\mathrm{Cl}, \mathrm{Br}$, $\mathrm{Cu}, \mathrm{Cd}$, and $\mathrm{Sn}$ ions. During isothermal heat treatments at $\mathrm{T}=600$ ${ }^{\circ} \mathrm{C}$, small liquid droplets of $\mathrm{CuX}$ precipitate resulting in $\mathrm{CuCl}_{0.4} \mathrm{Br}_{0.6}$ nanocrystals after cooling. It is known, that both $\mathrm{Cd}$ and $\mathrm{Sn}$ ions have a large influence on the photochromic properties and on the sizes of the nanocrystals. But the structural arrangement of the $\mathrm{Cd}$ and $\mathrm{Sn}$ ions in- or outside the nanocrystals is not known. In order to investigate the influence of $\mathrm{Cd}$ and $\mathrm{Sn}$ on the precipitation process and to understand the growth and growth delay processes, anomalous small angle X-ray scattering (ASAXS) experiments have been performed. The X-ray energy has been tuned near below the $\mathrm{K}$-absorption-edges of $\mathrm{Cu}, \mathrm{Br}, \mathrm{Cd}$ and $\mathrm{Sn}$ giving rise to a variation of the atomic scattering factor of the corresponding element. The result of the simultaneous fits of all curves assuming two different models for the particles will be presented. Both models, poly-disperse core-shell and diffusion zone surrounding spherical crystals, lead to the same conclusions. The crystalline core consists of the element $\mathrm{Cu}, \mathrm{Cl}$ and $\mathrm{Br}$. $\mathrm{Cd}$ and $\mathrm{Sn}$ are concentrated in a shell surrounding the nanocrystals. These structural models together with the measured viscoelastic behavior of the glass can explain the growth stop of the crystals after annealing of about $60 \mathrm{~min}$ at $600{ }^{\circ} \mathrm{C}$, knowing from previous SAXS studies.

Keywords: ASAXS, photochromic glasses, nanocrystals

\section{MS.78.1}

Acta Cryst. (2008). A64, C132

\section{Can amphipols be used to crystallize membrane proteins?}

Jean-Luc Popot, Delphine Charvolin, Martin Picard

Laboratoire de Physicochimie Moléculaire des Membranes Biologiques, UMR 7099, CNRS and Université Paris-7, Institut de Biologie PhysicoChimique, CNRS IFR 550, 13, rue Pierre et Marie Curie, F 75005 Paris, France.

'Amphipols' are a family of specially designed amphipathic polymers that can substitute to detergents at the hydrophobic transmembrane surface of membrane proteins $(1,2)$. Amphipol-trapped membrane proteins are soluble in aqueous solutions in the absence of detergent, in their native state, and, as a rule, much more stable than in detergent solutions (1-4). Because amphipols are very mild surfactants, they provide a favorable medium in which to fold to their native state denatured membrane proteins (5), including $G$ protein-coupled receptors overexpressed as inclusion bodies (6). Amphipol-trapped membrane proteins can be studied by NMR (7), electron microscopy (8), and most spectroscopic and other biophysical methods $(2,3)$. Because the protein/polymer association is irreversible, trapping with a functionalized amphipol will functionalize the protein without having to modify it chemically or genetically (9). Thus, trapping with a biotinylated amphipol makes it possible to attach the protein to a solid support for the purpose of screening for biological partners, ligands, drugs, antibodies etc. (10). Whether amphipols can be used to crystallize membrane proteins remains, however, an open question. An update will be presented of where we stand relative to this particular application and what the perspectives seem to be.

1. Tribet, C., Audebert, R. \& Popot, J.-L. (1996) Proc. Natl. Acad. Sci. USA 93, 15047-15050.

2. Popot, J.-L., et al. (2003) Cell. Mol. Life Sci. 60, 1559-1574.

3. Gohon, Y., et al. (2008) Biophys. J., 94, 3523-3537.

4. Picard, M., et al. (2006) Biochemistry 45, 1861-1869.

5. Pocanschi, C.L., et al. (2006) Biochemistry 45, 13954-13961.

6. Dahmane, T., et al. (2008) Submitted for publication.

7. Zoonens, M., et al. (2005) Proc. Natl. Acad. Sci. USA 102, 8893-8898.

8. Flötenmeyer, M., et al. (2007) J. Microsc. 227, 229-235.

9. Zoonens, M., et al. (2007) Biochemistry 46, 10392-10404.

10. Charvolin, D., et al. In preparation.

Keywords: membrane proteins, detergents, amphipols

\section{MS.78.2}

Acta Cryst. (2008). A64, C132-133

\section{Crystallisation of the calcium pump of skeletal muscle sarcoplasmic reticulum}

Chikashi Toyoshima

Institute of Molecular and Cellular Biosciences, The University of Tokyo, Tokyo, Japan, E-mail:ct@iam.u-tokyo.ac.jp 